\title{
CARCINOMATOSE PERITONEAL METASTÁTICO: UM CASO CONDUZIDO SEM A LOCALIZAÇÃO DO SÍTIO PRIMÁRIO DO TUMOR
}

\author{
METASTATIC PERITONEAL CARCINOMATOSIS: A CASE \\ CONDUCTED WITHOUT THE LOCATION OF THE TUMOR PRIMARY \\ SITE
}

\author{
Raphael Formiga Medeiros Maciel ${ }^{1}$ \\ Francisco Carlos Oliveira Júnior² \\ Igor de Sousa Gabriel ${ }^{3}$ \\ Ankilma do Nascimento Andrade Feitosa ${ }^{4}$
}

Resumo: Objetivo: relatar a terapêutica adotada em um caso de neoplasia peritoneal metastático que não obteve o sítio primário localizado por métodos convencionais diagnósticos. Metodologia: trata-se de um estudo do tipo descritivo, com abordagem qualitativa, baseado em estudo de caso, realizado a partir de pesquisa em prontuário como instrumento para coleta de dados. A pesquisa obedeceu aos princípios éticos, em conformidade com a resolução 466/2012, do Conselho Nacional de Saúde, que trata de pesquisa e testes envolvendo seres humanos. O projeto foi submetido e aprovado pelo Comitê de Ética em Pesquisa da Faculdade Santa Maria. Resultados: com um quadro clínico inicial bastante inespecífico foi necessário a realização de diversos exames, tanto laboratoriais quanto de imagem, para a investigação dos diagnósticos diferenciais, neoplásicos ou não, que poderiam acometer o peritônio, até se firmar o diagnóstico como carcinomatose peritoneal metastático, porém, sem a confirmação da origem da lesão. O caso foi conduzido com terapêutica direcionada para a metástase, enquanto continuava-se a realizar uma gama de exames para investigação do foco primário do tumor, o que poderia aumentar a eficácia do tratamento, desde que houvesse uma conduta mais direcionada ao foco principal da lesão tumoral. Conclusão: foi relatado o caso demonstrando que mesmo com uma longa jornada investigativa não se obteve desfecho diagnóstico. A principal hipótese aceita para o caso em questão foi a de tumor primário do próprio peritônio. Prolongou-se por

\footnotetext{
${ }^{1}$ Acadêmico do curso de Medicina da Faculdade Santa Maria, Cajazeiras - PB.

${ }_{2}^{2}$ Médico. Docente do curso de Medicina da Faculdade Santa Maria, Cajazeiras - PB.

${ }^{3}$ Médico. Docente do curso de Medicina da Faculdade Santa Maria, Cajazeiras - PB.

${ }^{4}$ Enfermeira. Docente do curso de Medicina da Faculdade Santa Maria, Cajazeiras - PB.
} 
pouco tempo a sobrevida do paciente, que veio a óbito cerca de 1 ano e 6 meses após o início da sintomatologia.

Palavras - chave: Carcinoma, Metástase Neoplásica, Peritônio.

\begin{abstract}
Objective: to report the therapy adopted in a case of metastatic peritoneal neoplasia that did not obtain the primary site located by conventional diagnostic methods. Methodology: this is a descriptive study, with a qualitative approach, based on a case study, carried out based on research in medical records as an instrument for data collection. The research followed the ethical principles, in accordance with resolution 466/2012, of the National Health Council, which deals with research and tests involving human beings. The project was submitted to and approved by the Research Ethics Committee of Faculdade Santa Maria. Results: with a very unspecific initial clinical picture, it was necessary to perform several tests, both laboratory and imaging, to investigate the differential diagnoses, neoplastic or not, that could affect the peritoneum, until the diagnosis was established as metastatic peritoneal carcinomatosis, however, without confirmation of the origin of the injury. The case was conducted with therapy aimed at metastasis, while a range of tests to investigate the primary focus of the tumor continued, which could increase the effectiveness of the treatment, as long as there was a more focused approach to the main focus of the lesion tumor. Conclusion: The case was reported demonstrating that even with a long investigative journey there was no diagnostic outcome. The main hypothesis accepted for the case in question was that of a primary tumor of the peritoneum itself. The patient's survival was briefly prolonged, and he died about 1 year and 6 months after the onset of symptoms.
\end{abstract}

Keywords: Carcinoma, Neoplasm metastasis, Peritoneum. 


\section{INTRODUÇÃO}

A carcinomatose peritoneal (CP) é o câncer generalizado no peritônio que pode ser classificado como primário, quando é proveniente do próprio peritônio, ou secundário. A grande maioria são tumores secundários (metastáticos) oriundos de carcinoma colorretal, pancreático, gástrico, do ovário e do apêndice cecal. Já as lesões primárias são mais raras, elas começam no próprio peritônio, e incluem o carcinoma primário do peritônio e o mesotelioma (LOPES; BRESSAN, 2013).

O carcinoma primário do peritônio foi descrito pela primeira vez em 1959, com uma incidência de 6,78 casos por milhão de habitantes. Os sintomas mais comuns deste tipo de carcinoma proximal são: comprometimento do estado geral com perda de peso, desconforto abdominal, dor abdomino-pélvica e distensão abdominal devido a dano peritoneal difuso. $O$ achado mais comum é a ascite, relatada em $85 \%$ dos casos (SAINT-PIERRE et al., 2015).

O desenvolvimento de um implante peritoneal ocorre em duas etapas, que são: a primeira pela libertação de células tumorais para a cavidade abdominal através da manipulação do tumor, dos canais linfáticos ou de vasos sanguíneos; e a segunda pelo aprisionamento delas pelo processo inflamatório, em que as células tumorais ficam aderidas na fibrina, causando um aumento de células inflamatórias e fatores de crescimento que favorecem a formação de um implante metastático no peritônio (CERONI V. et al., 2014).

A CP está associada a um péssimo prognóstico, pois o tratamento curativo ideal costuma ser difícil, por isso ao estudá-la deve-se sempre considerar alguns diagnósticos diferenciais (VAN BAAL et al., 2017). A Tuberculose Peritoneal (TP), que é uma forma extrapulmonar de apresentação da tuberculose, é um dos principais diagnósticos diferenciais da CP. Esse diagnóstico requer confirmação microbiológica ou histopatológica, através da biópsia peritoneal com coleta de amostras para estudos, incluindo micobactérias. Além de apoiar estudos de imagem 
radiológica como a ultrassonografia abdominal e a tomografia computadorizada (OSPINA-MORENO et al., 2014).

Além da TP, existem variados tipos de câncer que podem metastizar para o peritônio (LOPES; BRESSAN, 2013). Estudos mostram que é infrequente o aparecimento de carcinomatose peritoneal secundária ao carcinoma de mama e que, quando aparece, costuma estar associada ao carcinoma lobular infiltrante. Os sintomas são inespecíficos e podem aparecer vários anos após a detecção do tumor primário, o que dificulta a diferenciação entre metástase de carcinoma de mama e tumor primário da cavidade peritoneal (PRIEGO J et al., 2007).

O câncer gástrico também é de importante relevância na investigação, devido haver uma grande chance de cura em estágios iniciais, quando o achado endoscópico ainda é um tumor incipiente, porém, tem alta taxa de mortalidade quando diagnosticado em estágios avançados. Nestes casos, o tratamento com intenção curativa tem alta taxa de recorrência peritoneal, podendo chegar até $50 \%$ (CERONI V. et al., 2014).

Outro tipo de câncer que frequentemente acomete o peritônio é o Carcinoma Colorretal (CCR), sendo o terceiro tipo de câncer mais comum nos homens, com uma estimativa aproximada de $40 \%$ de disseminação ocasionando a CP. Em 25\% dos pacientes com CP, a metástase fica restrita a cavidade peritoneal e, em cerca de $30 \%$ dos pacientes, ela vai ser a causa principal da morte dos pacientes com CCR (MOSCA et al., 2017).

Sendo a neoplasia uma patologia bastante agressiva e de prognóstico reservado, justifica-se a realização desse estudo para nortear os pesquisadores em saúde acerca do tema, expandido as perspectivas futuras a respeito do diagnóstico e da condução de novos casos, de modo a auxiliar os profissionais da área, visando redução das complicações e os níveis de morbimortalidade.

As informações contidas neste trabalho foram obtidas através de estudo do prontuário, contendo os laudos dos exames dos métodos diagnósticos e a conduta as quais o paciente foi submetido, além de revisão da literatura. Assim, o presente estudo tem por objetivo relatar a terapêutica adotada em um caso de neoplasia peritoneal metastático que não obteve o sítio primário localizado por métodos convencionais diagnósticos. 


\section{METODO}

O presente estudo caracteriza-se como uma pesquisa do tipo descritiva, com abordagem qualitativa, baseado em estudo de caso que inclui um único indivíduo adulto, do sexo masculino, falecido aos 60 anos de idade, portador de carcinomatose peritoneal metastático.

O estudo foi autorizado pelo Centro Paraibano de Oncologia (CPO), localizado na cidade de João Pessoa-PB, responsável pelos documentos e exames do paciente portador de carcinomatose peritoneal metastático. Foi realizada a coleta de dados por meio de estudo em prontuário contendo as informações sobre a história clínica com a sintomatologia, laudos dos exames realizados (laboratoriais, biopsias contentando os resultados histopatológicos e exames de imagem como a ultrassonografia, tomografia computadorizada e a tomografia por emissão de pósitrons - PET-scan) durante todo o processo diagnóstico e as medicações utilizadas na terapêutica.

Esta pesquisa está de acordo com as normas éticas e as diretrizes promovidas na Resolução N 466/2012 do Conselho Nacional de Saúde/Ministério da Saúde (CNS/MS), que regulamentam as pesquisas que envolvem seres humanos no país. O trabalho foi encaminhado e aprovado pelo Comitê de Ética em Pesquisa (CEP) da Faculdade Santa Maria, localizada no município de Cajazeiras - PB.

\section{RELATO DO CASO}

Paciente do sexo masculino, 59 anos, médico, hipertenso, sem outras comorbidades. Com histórico de um caso de câncer gástrico na família. Iniciou a sintomatologia em meados de março de 2014, com queixas de fortes dores em região lombar bilateral, com irradiação para região inguinal, realizou exame de 
imagem das vias urinarias que evidenciou nefrolitíase bilateral. Seguiu em conduta conservadora com aumento de ingesta hídrica e anti-inflamatórios não esteroidais.

No início do mês de abril de 2014 expeliu o cálculo evidenciado nos exames de imagem, porém, continuou sentindo incômodo abdominal. Deu sequência à investigação com exames de imagem do abdome total que evidenciaram processo inflamatório peritoneal, levantando-se as hipóteses diagnósticas de tuberculose peritoneal ou carcinomatose peritoneal.

Continuou a investigação diagnóstica em maio de 2014, com culturas do líquido e fragmentos peritoneais, sendo descartado crescimento bacteriano ou infecção fúngica, entretanto evidenciou um quadro citológico positivo para células neoplásicas.

Em junho de 2014 realizou o primeiro PET-scan e novos exames do líquido peritoneal que fecharam o diagnóstico como carcinomatose metastática. A partir disso, já iniciou o tratamento quimioterápico com o protocolo mFOLFOX6, com sessões quinzenais, associado a medicações sintomáticas e acompanhado de exames laboratoriais de controle.

Logo após, iniciou-se a investigação para pesquisa do foco primário do tumor. Foi identificado uma pangastrite enantematosa com lesões polipoides em antro. Realizado mucosectomia endoscópica, hemostasia endoscópica com Hemoclip e tatuagem endoscópica após a ressecção com tinta Nanquim. Foram sugeridas então duas possíveis fontes para lesão primária, o trato biliopancreático ou o trato gastrointestinal/apêndice cecal.

Encontrou-se, ainda no primeiro PET-scan, uma opacidade pulmonar discretamente hipermetabólica, aparentemente inflamatória/infecciosa, mas que houve resolução, tendo em vista que no segundo PET-scan, realizado em agosto de 2014, não foi mais visualizado essa alteração, entretanto, percebeu o surgimento de nódulo pulmonar no lobo superior esquerdo que poderia estar relacionado a doença secundária. Isso demonstra uma resposta parcial significativa a terapia instituída.

No terceiro PET-scan, realizado em outubro de 2014, houve evidência de resposta metabólica completa na carcinomatose peritoneal/omental, persistindo apenas densificação a tomografia. O nódulo pulmonar no segmento ápico-posterior do lobo superior esquerdo, surgido no exame anterior, alterou sua morfologia, agora 
notando-se opacidades algo nodulariformes com sinais sugestivos de disseminação endobrônquica, que pode estar relacionado a obliteração das vias aéreas distais, sugerindo um processo inflamatório/infeccioso ou impactação mucoide.

Encerrou a primeira etapa do tratamento quimioterápico por volta de outubro de 2014, na qual fez uma paralisação na medicação AVASTIN para realização de procedimento cirúrgico, programado para ser realizado no início de fevereiro. Já em Tomografia Computadorizada (TC) de tórax, realizada em novembro de 2014, apresentou uma extensão um pouco menor do que o exame anterior, o que sugeriu bronquiloectasias ou impactação mucoide.

Adentrando em 2015, foi realizado, em janeiro, o quarto PET-scan, que identificou um tumor viável com aumento do metabolismo glicolítico em carcinomatose peritoneal extensa e na cauda do pâncreas. Nessa época o paciente voltou a sentir desconforto abdominal e adentrou o esquema quimioterápico do protocolo FOLFIRI.

Em fevereiro de 2015 fez o procedimento cirúrgico por videolaparoscopia para retirada da lesão, porém, não foi possível devido recidiva da lesão com disseminação em abdome. No anátomo patológico do epíplon foi confirmado o adenocarcinoma infiltrando o tecido conjuntivo adiposo, corroborado com a citologia aspirativa de líquido ascítico que demonstrou adenocarcinoma metastático.

Logo no início de março de 2015 retomou o esquema terapêutico com o AVASTIN, e seguiu queixando-se de dores abdominais e constipação intensa, realizou exames no qual foi evidenciado líquido ascítico aumentado e aumento no trânsito intestinal, sem sinais de dilatação de alças, com meteorismo aumentado no colón direito.

Em TC do abdome total, sem contraste, foi visualizado uma acentuada dilatação do intestino grosso, com moderado acúmulo de resíduo fecal intraluminal observando-se área de redução do calibre ao nível do colón sigmoide, podendo corresponder a suboclusão mecânica. Foi submetido, então, a laparotomia exploradora para desobstrução intestinal, na qual foi realizado desvio do trato gastrointestinal por ileostomia.

No quinto PET-scan, realizado em abril de 2015, evidenciou-se a persistência no aumento do metabolismo glicolítico, em extensa carcinomatose peritoneal e em 
lesão em relação com a cauda do pâncreas, devendo corresponder a tecido neoplásico viável. Foi suspenso o AVASTIN e associado ao esquema terapêutico o ERBITUX. Ao final do mês, apareceram sinais de obstrução intestinal alta com má digestão, queixando-se de "empachamento" e, em maio de 2015, passou a apresentar vômitos refratários a medicação antiemética.

Foi internado em ambiente hospitalar no início de junho de 2015 para iniciar nutrição parenteral através de acesso venoso de grande calibre, sendo acompanhado pela equipe multiprofissional do estabelecimento. Com uma decaída no estado clínico geral, apresentou aumento considerável do líquido ascítico, sendo necessária a realização de paracentese de alívio.

Evoluiu com desnutrição, entrando em estado caquético e anasarca, passou a ficar acamado e apresentar delirium. A partir disso foi aplicado o último esquema com o protocolo GEMOX e, logo após, decidido em conjunto pelo seguimento paliativo, tendo em vista que o paciente estava em deterioramento clínico e não mais tinha condições de tratamento quimioterápico.

Em 09 de agosto de 2015, o paciente apresentou piora considerável com falência múltipla de órgãos, apresentando letargia, anúria, má circulação periférica e respiração agônica, tendo o óbito declarado por volta das 13 horas daquele dia pelo médico clínico plantonista.

\section{RESULTADOS E DISCUSSÕES}

A sintomatologia do paciente iniciou de forma inespecífica, com quadro de nefrolitíase, o qual, ao expelir o cálculo, percebeu novos sintomas que levaram a uma sequência de investigação diagnóstica. Suspeitou-se de $\mathrm{CP}$, que é uma doença de etiologia indeterminada, definida como a disseminação e implante de células neoplásicas na cavidade peritoneal. Pelos primeiros exames realizados foi suspeitado também de tuberculose (TB) entero-peritoneal, que é o principal diagnóstico diferencial não tumoral da Carcinomatose Peritoneal (NAKANDAKARI et al., 2015). 
A TB é responsável por 1,3 milhões de mortes registradas em todo o mundo, sendo a segunda principal causa de morte por doenças infecciosas. A tuberculose peritoneal é uma forma pouco frequente de TB extrapulmonar, responsável por até 10\% dos casos de tuberculose extrapulmonar (TOMÁS et al., 2017).

É uma doença muito frequente nos países subdesenvolvidos, especialmente nas áreas mais povoadas. Segundo a Organização Mundial da Saúde (OMS), no mundo alguém é infectado com tuberculose a cada segundo e, aproximadamente, a cada ano, $1 \%$ da população mundial é reinfectada. Porém, nem todas as pessoas infectadas desenvolvem a doença na forma ativa, apenas 5 a 10\% manifestarão os sintomas (FLORES et al., 2017).

Quando os bacilos conseguem vencer as defesas respiratórias e alcançam os alvéolos e linfonodos, desencadeia-se a tuberculose disseminada, que também pode ir para os demais órgãos através da via hematogênica. Podendo provocar lesões localizadas ou múltiplas a depender da resposta do sistema imunológico (PEREIRA; MACHADO; GERECHT, 2008).

Esta doença geralmente tem sintomas inespecíficos, que leva a um diagnóstico tardio mesmo quando o grau de suspeição é alto. Isso tem sido atribuído a vários fatores, incluindo apresentação clínica inespecífica ou dificuldade em obter amostras teciduais para diagnóstico, consequentemente o atraso no diagnóstico e tratamento estão associados a pior prognóstico (TOMÁS et al., 2017).

No peritônio, a assiduidade de sua afetação contrasta com a dificuldade de encontrar os ferimentos, geralmente mascarados por aderências. O estudo histológico pode revelar diferentes tipos de lesões. Assim como a demonstração, geralmente por cultura, da presença de Mycobacterium. Sua negatividade não exclui o diagnóstico. Deve ser insistido na cultura quantas vezes forem necessárias, desde que a história, a clínica, o curso, a radiologia e laparotomia apontam para o diagnóstico de tuberculose (FLORES et al., 2017).

O diagnóstico de TB extrapulmonar foi descartado após exames de imagem e citologia do líquido peritoneal, que confirmaram o anatomopatológico de CP. É considerada como CP secundária a disseminação intraperitoneal de um tumor que não tem origem no próprio peritônio. É uma doença maligna de péssimo prognóstico, 
geralmente indica uma fase terminal, e é a doença peritoneal difusa mais comum (SILVA et al., 2016).

De acordo com NAKANDAKARI et al., (2015) a CP pode cursar com dor abdominal difusa, intensa, que não cede com a mudança de posição ou com o uso de analgésicos. Podendo ainda apresentar constipação, náuseas e/ou vômitos e ausência de flatulência. O que corrobora com ALVES (2012) que relata que os resultados do exame físico na $\mathrm{CP}$ são bastante inespecíficos, sendo os mais frequentes a obstrução intestinal e a ascite.

MOSCA et al. (2017) relatam que a disseminação intra-celômica pode ocorrer por inúmeros processos, que podem ou não ser decorrentes de trauma cirúrgico. $A$ CP é sempre indicativa de estágio oncológico avançado. Geralmente ocorre em casos de câncer de ovário, estômago, cólon e pâncreas, sendo menos frequentemente em hepato-celular, renal, uterino, bexiga, linfoma e tumores de origem desconhecida (NAKANDAKARI et al., 2015).

A contaminação metastática ocorre pela livre circulação de células neoplásicas na cavidade peritoneal, formando múltiplos nódulos neoplásicos nesta cavidade. Apesar da CP ocorrer através de uma disseminação sistêmica, há benefícios comprovados do uso da terapia locoregional, sendo possível abordar a CP como uma progressão loco-regional do tumor primário (ALVES, 2012).

Também é importante investigar outros possíveis focos de disseminação metastática como diagnósticos diferenciais. O câncer maligno de mama é o mais comum nas mulheres, porém, é raro em homens, assim como a carcinomatose peritoneal por câncer de mama, sendo responsável por 6 a 8\% dos casos de adenocarcinoma de mama. O tipo lobular da doença está mais associado a metástases no trato gastrointestinal, nos órgãos genitais e no peritônio (BALSAMO et al., 2012).

O estudo de BALSAMO et al. (2012) comenta ainda que nos casos de adenocarcinoma de mama que evoluam com carcinomatose peritoneal, é essencial a investigação da origem da carcinomatose, pois um bom alívio pode ser obtido com um tratamento específico. A quimioterapia, associada a cirurgias conservadoras, também pode proporcionar boa qualidade de vida. O diagnóstico dessa entidade é 
essencial para o oncologista, uma vez que casos de remissão da doença foram descritos com a quimio e hormônio terapia adequadas (PRIEGO J et al., 2007).

O câncer gástrico é considerado como a quarta maior malignidade em todo o mundo, o que o leva a fazer parte da investigação diferencial. Mesmo quando a ressecção cirúrgica é possível, a sobrevida em longo prazo é observada apenas na minoria dos pacientes, com sobrevida global em cinco anos menor que 30\%. O fator prognóstico mais importante que influencia a sobrevida de pacientes com câncer de estômago é a extensão da doença avaliada pelo estágio do tumor (HOSKOVEC et al., 2012).

Os protocolos tradicionais do acompanhamento no pós-operatório do carcinoma gástrico geralmente diagnosticam a doença em estágio avançado, o que causa uma drástica redução nas chances curativas nestes pacientes, o que torna sombrio o prognóstico do carcinoma recorrente, com uma sobrevida de no máximo um ano (CREPALDI-FILHO et al., 2008).

A disseminação peritoneal é o padrão mais comum de recidiva no câncer gástrico, mesmo após uma ressecção potencialmente curativa. Essa característica pode ser atribuída à possível disseminação intraperitoneal de células malignas já presentes no momento da cirurgia ou a manipulações cirúrgicas. Além disso, uma alta taxa de recorrência é indicativa de um mau prognóstico e estágios oncológicos avançados (TUSTUMI et al., 2016).

A investigação do Carcinoma Colorretal (CCR) é de suma importância, já que de acordo com MOSCA et al. (2017), a disseminação inicial do CCR pode ocorrer através de três vias, que são a via linfática, hematogênica ou metastização peritoneal. A contaminação peritoneal pode apresentar lesões superficiais do peritônio parietal e visceral.

Aproximadamente $20 \%$ dos pacientes portadores de câncer colorretal se apresentam, no momento do diagnóstico, no estádio IV, menos de $20 \%$ deles são candidatos para uma ressecção cirúrgica potencialmente curativa, com um prognóstico reservado a uma expectativa global que não excede $5 \%$ em cinco anos (BANNURA C. et al., 2009).

MOSCA et al. (2017) continua explicando que a CP pode ocorrer de forma espontânea como complicação natural da doença ou pode estar relacionado ao 
implante no momento cirúrgico. A cirurgia pode ter um resultado inesperado de péssimo prognostico, caso converta um processo localizado em doença metastática.

Devido a gama de possibilidades de tumores que podem metastizar para o peritônio, é extremamente necessário o correto diagnóstico do foco para uma adequada condução terapêutica. ALVES (2012) afirma que a PET-scan e a TC podem ser importantes para o diagnóstico da $\mathrm{CP}$, apesar de que com os métodos de imagens atuais é difícil diagnosticar a carcinomatose e sua extensão na cavidade. Já RIBEIRO-JÚNIOR et al., (2010) relatam que, através da ultrassonografia ou TC, é difícil obter dados fidedignos do envolvimento do mesentério e do intestino delgado.

A videolaparoscopia é um excelente método para o estadiamento e exérese de tumores malignos, pois permite uma ampla exploração anatômica, com uma taxa de eficiência entre 90 e 100\%. Também podem ser solicitados o lavado peritoneal (com correto diagnóstico entre 70 a 90\%) para realização do citológico, coleta do líquido ascítico e biopsia com histopatológico da lesão suspeita (RIBEIRO-JÚNIOR et al., 2010).

O estudo do líquido ascítico é importante no diagnóstico da carcinomatose peritoneal pois pode evidenciar células neoplásicas (ALVES, 2012). Marcadores tumorais também são valiosos para o planejamento sobre a indicação de terapias adjuvantes, principalmente após ressecção curativa de carcinoma avançado (CREPALDI-FILHO et al., 2008).

O peritônio é uma monocamada de células mesoteliais em que os vasos sanguíneos estão distantes dessa camada, essa seria uma das razões pela qual a quimioterapia sistêmica não tenha eficácia suficiente para que haja uma regressão completa dos implantes peritoneais, ou evite a recorrência peritoneal. Para conseguir atingir o peritônio e obter algum grau de resposta, teria que ser utilizada doses de quimioterapia consideradas tóxicas. Por isso, é importante pensar em tratamentos adjuvantes através da cavidade peritoneal, em que a passagem da quimioterapia para a circulação sistêmica é mínima (CERONI V. et al., 2014).

ALVES (2012) relata que a carcinomatose peritoneal faz parte das neoplasias de estágio avançado, com péssimo prognóstico, cuja única opção terapêutica era o tratamento paliativo e de suporte. Porém, nas últimas décadas surgiram novas perspectivas com armas terapêuticas como a quimioterapia intraperitoneal e a 
cirurgia de citoredução, com resultados promissores a depender da origem do tumor primário.

A quimioterapia intraperitoneal quando comparada ao tratamento sistêmico, tem se mostrado mais benéfica devido a ação direta dos medicamentos na cavidade intraperitoneal, com melhor eficácia sob as lesões disseminadas e redução da toxicidade do efeito sistêmico. Em pacientes com metástase intraperitoneal, a quimioterapia sistêmica não tem benefícios resolutivos, será apenas um meio paliativo para prolongar a sobrevida do doente. Porém, para pacientes com carcinomatose peritoneal não se observou tal sobrevida, pacientes com doença peritoneal continuaram a morrer poucos meses após o diagnóstico (SEITENFUS et al., 2018).

\section{CONCLUSÃO}

Foi relatado o caso demonstrando que mesmo com uma longa jornada investigativa não se obteve desfecho diagnóstico. A principal hipótese aceita para o caso em questão foi a de tumor primário do próprio peritônio, que não obteve grandes resultados com condução terapêutica quimioterápica. Prolongou-se por pouco tempo a sobrevida do paciente, que veio a óbito cerca de 1 ano e 6 meses após o início da sintomatologia.

Como visto há uma dificuldade em relação a identificação inicial da lesão, em virtude da diversidade de diagnósticos a serem investigados, o que possivelmente pode prorrogar o início do tratamento adequado. Concluindo assim que se faz necessário a realização de mais pesquisas acerca das neoplasias, em especial a $\mathrm{CP}$, visando o aprimoramento das técnicas utilizadas no diagnóstico para que possam promover a terapêutica ideal de modo precoce. 


\section{REFERÊNCIAS BIBLIOGRÁFICAS}

ALVES, C.M.F.P. Carcinomatose peritoneal de neoplasias do tubo digestivo. Revista Portuguesa de Cirurgia, Faculdade de Medicina Universidade do Porto, 2012, 13 set. 2012.

BALSAMO, F. et al. Intestinal obstruction due to malign breast neoplasm and peritoneal carcinomatosis: A case report. Journal of Coloproctology, v. 32, n. 2, p. 188-192, 2012.

BANNURA C., G. et al. Rol de la resección paliativa en cáncer colorrectal estadio iv: Análisis de 177 pacientes. Revista Chilena de Cirugia, v. 61, n. 3, p. 266-274, 2009.

CERONI V., M. et al. Prevención de la recurrencia peritoneal en el tratamiento quirúrgico del cáncer gástrico. Revista Chilena de Cirugia, v. 66, n. 3, p. 283-291, 2014.

CREPALDI-FILHO, R. et al. Levels of carcinoembryonic antigen and CA 19-9 in the sera and peritoneal washing of patients undergoing surgical treatment for gastric carcinoma. Arquivos de Gastroenterologia, v. 45, n. 3, p. 219-224, 2008.

FLORES, S. E. H. et al. Tuberculosis pélvica y antecedente de embarazo. Reporte de caso. Revista de la Facultad de Medicina (México), v. 60, n. 1, p. 17-22, 2017.

HOSKOVEC, D. et al. Levels of CEA and Ca 19 - 9 in the sera and peritoneal cavity in patients with gastric and pancreatic cancers. Acta Cirurgica Brasileira, v. 27, n. 6, p. 410-416, 2012.

LOPES, A.; BRESSAN, A.K. Tratamento da Carcinomatose Peritoneal. In: HOFF, P.M.G.et al. Tratado de Oncologia. São Paulo: Atheneu, 2013. cap. 127, p. 1805-1820.

MOSCA, et al. Abordagem Terapêutica da Carcinomatose Peritoneal em doentes com Carcinoma Colorretal. Revista Portuguesa de Cirurgia, [S.L], v. 2, n. 40, jun. 2017.

NAKANDAKARI, M. D. et al. Carcinomatosis peritoneal secundaria a adenocarcinoma de células en anillo de sello del colon: A propósito de un caso. Revista Medica Herediana, v. 26, n. 3, p. 190, 2015.

OSPINA-MORENO, C. et al. Peritoneal tuberculosis. Radiographic diagnosis. Revista Espanola de Enfermedades Digestivas, v. 106, n. 8, p. 548-551, 2014.

PEREIRA, J.C.B.; PEREIRA, R.R.M.; GERECHT, S.A. Tuberculose disseminada - Caso clínico e discussão. Revista Portuguesa Pneumologia, [S.L], v. 14, n. 4, p. 561-569, jul./ago. 2008.

PRIEGO J, P. et al. Carcinomatosis peritoneal secundaria a carcinoma Lobulillar de mama. Revista chilena de cirugía, v. 59, n. 3, p. 3-8, 2007.

RIBEIRO-JÚNIOR, U. et al. Método laparoscópico no diagnóstico e extensão da carcinomatose peritoneal. ABCD. Arquivos Brasileiros de Cirurgia Digestiva (São Paulo), v. 23, n. 3, p. 196-199, 2010.

SAINT-PIERRE, G. et al. Carcinoma Seroso Papilar Peritoneal Primario: serie de casos Hospital Clínico Universidad de Chile 2010-2015. Revista Científica Ciencia Médica, v. 18, n. 2, p. 1417, 2015.

SEITENFUS, R. et al. Pressurized intraperitoneal aerosol chemotherapy (PIPAC) through a single port: Alternative delivery for the control of peritoneal metastases. Revista do Colegio Brasileiro de Cirurgioes, v. 45, n. 4, p. 1-8, 2018. 
SILVA, B.R. et al. Peritonite granulomatosa causada por fibras vegetais imitando carcinomatose peritoneal. Revista Portuguesa de Cirurgia, Serviço de Cirurgia Geral - Hospital Pedro Hispano, ano 2016, v. 38, p. (38):33-40, 21 jun. 2016.

TOMÁS, C. et al. Peritoneal tuberculosis: diagnostic challenge for the gynecologist. Acta Obstétrica e Ginecológica Portuguesa, v. 11, n. 1, p. 61-63, 2017.

TUSTUMI, F. et al. Detection value of free cancer cells in peritoneal washing in gastric cancer: A systematic review and meta-analysis. Clinics, v. 71, n. 12, p. 733-745, 2016.

VAN BAAL, J. O. A. M. et al. The histophysiology and pathophysiology of the peritoneum. Tissue and Cell, v. 49, n. 1, p. 95-105, 2017. 